\title{
Study on the Characteristic of Adsorption Kinetics of Organochlorine Pesticides in Agricultural Soils
}

\author{
Ming GAO \\ Department of Environmental Science \\ Jilin University \\ Changchun Institute of Technology \\ Changchun, 130012, China \\ e-mail: sh_gm@ccit.edu.cn
}

\author{
Xuming SUN \\ Baicheng Local Maritime Bureau \\ Baicheng, 137000, China \\ e-mail: 3039679@qq.com
}

\author{
Hui HUANG \\ Baicheng Local Maritime Bureau \\ Baicheng, 137000, China
}

\begin{abstract}
To study the characteristic of adsorption kinetics of BHC and DDT in the unsaturated zone of typical northeastern soil field of China by adopting indoor soil adsorption simulation experiment. The purpose of the study is to reveal the pollution mechanism of BHC and DDT to soil and groundwater and provide a scientific basis for making reasonable and effective groundwater pollution prevention and control measures. The results showed that the adsorption of BHC and DDT in the tested soils were well consistent with the Langmuir kinetics equation. Adsorption can be divided into two processes, namely, rapid reaction phase and slow reaction phase. At the initial stage of reaction, the amount of adsorption increased with time and basically reached equilibrium at about 4 8 $\mathrm{h}$. The equilibrium adsorption capacity was more than $50 \%$ in the first hour and above $90 \%$ in the second hour. In addition, the sorption and the desorption process actually occur simultaneously during the adsorption or desorption of organic matter. The adsorption / desorption two-compartment model can describe the two reversible processes at the same time. The fitted value $r$ is from 0.8039 to 0.9651 .
\end{abstract}

Keywords-BHC; DDT; soil; adsorption kinetics

\section{INTRODUCTION}

\section{A. Producing Hardcopy Using Ms-Word}

In recent years, the large areas of soil pollution caused by using and accumulation of organochlorine pesticides has attracted wide attention. Soil organic pollution is hidden and lagging. Once it has accumulated in water body and soil, it would be hard to be degraded. It would be absorbed by crops before it along with food chain gets into human body. It has become such a pollutant worthy our concern that could be greatly hazardous to human health. Absorption-desorption process is deemed as a major chemical reaction in controlling over persistent OCP's performance. The absorption-desorption of chlorinated organic compounds mostly depends on its physical and chemical properties. As to OCP, due to its typically low aqueous solubility and high n-octanol-water partition coefficient, large portion of OCP existing in environment are distributed into organic matter of sediment or soil.
Usually, OCP's soil pollution is relevant to underground water pollution. Once soil be polluted by OCP, as time goes on, OCP would be leaching via rainfall and self-loaded water, and gradually transfer into underground water, then spread out along with flowing water, leading to contamination to underground water. This article select the farmland soil collected in China's northeastern industrial polluted area as the research object, to investigate soil's absorptive characteristics to BHC and DDT. The purpose is to obtain data through soil's absorption-desorption of BHC and DDT experiment, in an effort to more comprehensively learn the performance of soil's absorption-desorption of BHC and DDT, to provide basis for soil restoration and research on mechanism of underground water pollution.

\section{EXPERIMENTS}

\section{A. Soil and Reagent for Experiment}

The soil samples were collected from a nearby chemical plant in the northeastern region $\left(42^{\circ} 04^{\prime} 001 \mathrm{~N}, 123^{\circ} 29^{\prime} 352\right.$ E) and farmland near the industrial wastewater-contaminated river $\left(43^{\circ} 53^{\prime} 503 \mathrm{~N}, 125^{\circ} 05^{\prime} 839 \mathrm{E}\right.$ ) (denoted by $\mathrm{Z}$ and $\mathrm{L}$, respectively). Soil samples were collected in the 0-20, 20-40 and $40-60 \mathrm{~cm}$ deepth of the land according to the soil natural level. After the samples were taken back to the laboratory, they were fully air-dried, ground and sieved through a nylon sieve with a pore size of $2 \mathrm{~mm}$ for experiment.

The pure organochlorine pesticides used in this study were purchased from Germany Dr.Ehrenstorfer company. Purity of BHC, $\gamma$-BHC and p, p'-DDE is $98.3 \%, 98.5 \%$ and $98.5 \%$ respectively. The water used in the experiment was secondary deionized water.

DDT standard sample (Ministry of Agriculture Environmental Protection Research Institute): the concentration of p, p'-DDT is $100 \mu \mathrm{g} / \mathrm{ml}$, the medium is petroleum ether. BHC standard sample (Ministry of Agriculture Environmental Protection Research Institute): $\alpha$ BHC, $\gamma$-BHC, with concentration of $100 \mu \mathrm{g} / \mathrm{ml}$, the medium is petroleum ether.

Stock solution: $1 \mathrm{ml}$ of each standard sample dissolved in petroleum ether, then volume to $100 \mathrm{ml}$ in the volumetric 
flask. The basic physical and chemical parameters of soil and the content in Table I.

TABLE I. THE BASIC PHYSICAL AND CHEMICAL PARAMETERS OF THE TESTED SOIL

\begin{tabular}{|c|c|c|c|c|c|c|}
\hline No & L-1 & L-2 & L-3 & Z-1 & Z-2 & Z-3 \\
\hline depth(cm) & $1-20$ & $: 0-40$ & $.0-60$ & :0-40 & $0-40$ & $0-60$ \\
\hline $\mathrm{pH}$ & .70 & 6.18 & 5.88 & 5.06 & 6.02 & 4.63 \\
\hline TOC $(\%)$ & 1.611 & 1.437 & 0.539 & 2.199 & 1.802 & 1.933 \\
\hline $\mathrm{BD}(\mathrm{g} / \mathrm{cm} 3)$ & 1.346 & 1.336 & 1.326 & 1.668 & 1.503 & 1.412 \\
\hline $\mathrm{EC}(\mu \mathrm{s} / \mathrm{cm})$ & 28 & 42 & 51 & 88 & 102 & 128 \\
\hline $\mathrm{CEC}(\mathrm{mmol} / \mathrm{kg}$ & 157.0 & 160.0 & 137.2 & 157.9 & 153.2 & 164.8 \\
\hline ) & 6 & 0 & 4 & 9 & 4 & 3 \\
\hline Sand soil (\%) & 18 & 26 & 38 & 16 & 21 & 35 \\
\hline Silt (\%) & 58 & 52 & 45 & 61 & 59 & 48 \\
\hline clay $(\%)$ & 24 & 22 & 17 & 23 & 20 & 17 \\
\hline
\end{tabular}

\section{B. Experimental Methods}

Preparation of BHC and DDT solution: Weigh a certain amount of BHC and DDT separately, dissolve in $100 \mathrm{ml}$ volumetric flask with methanol, and then rinse the beaker and glass rod twice with a certain amount of methanol, pour the rinse into a volumetric flask, then to volume by methanol, finally pour into the a glass vial with a pad cap for short-term storage.

Dilute the stock solution to $50 \mu \mathrm{g} / \mathrm{L}$, then use the liquid extraction and solid phase extraction methods for sample pretreatment respectively. Of which solid phase extraction used different activators and eluents to get different recovery effect. According to the recovery rate the method of extracting organic chlorine was chosen in the sample which was quickly, easily, and less-consumpted, making the organochlorine pesticide in the water sample be completely extracted as far as possible.

$50 \mathrm{ml}$ of the solution was selected, and then a certain volume of BHC and DDT standard stock solution was added to form a series of adsorbent liquid with the concentration of 1.0 and $12.5 \mu \mathrm{g} / \mathrm{L}$ respectively. The $2.5 \mathrm{~g}$ two handled $\mathrm{Z}, \mathrm{L}$ soils were accurately weighed and added into the above solution in the conical flask, and then numbered them. And then they were shaking in the shaker for continuous $16 \mathrm{~h}$ for adsorption experiments at the room temperature. A conical flask was taken and centrifuged at 2, 5, 15, 30, 60, 120, 240, $480,960,1440,2400 \mathrm{~min}$ each time, the exact amount of $40 \mathrm{ml}$ solution was taken from each for enrichment by solid phase extraction. Using cyclohexane as eluent, after elution nitrogen blown concentrated to $2 \mathrm{ml}$, transferred to a glass sample vial with the screw pad cap to save, and measure the content of BHC and DDT.

\section{RESUlTS AND DisCUSSION}

Langmuir kinetic equation is a commonly used empirical model to describe the adsorption process in solution. The Langmuir kinetic equation is used to describe non-linear experimental data by calculating the parameters. The Langmuir kinetic equation is as in $\mathrm{Eq}(1)$ :

$$
C_{S}=\frac{K_{1} \cdot C_{S}{ }^{\max } \cdot t}{1+K_{1} t}
$$

of the formula, $\mathrm{t}$ is the adsorption time (h), Cs is the organochlorine pesticide adsorption amount $(\mu \mathrm{g} / \mathrm{g})$ in the soil at time of $\mathrm{t}, \mathrm{Csmax}$ is the equilibrium adsorption capacity of the organochlorine pesticide $(\mu \mathrm{g} / \mathrm{g}), \mathrm{K} 1$ is Langmuir Kinetic constant (1/h). Figure1, 2, and 3 are the changes of unit fitted adsorption capacity of $\alpha-\mathrm{BHC}, \gamma-\mathrm{BHC}$ and $\mathrm{p}, \mathrm{p}$-DDT in soils $\mathrm{Z}$ and $\mathrm{L}$.
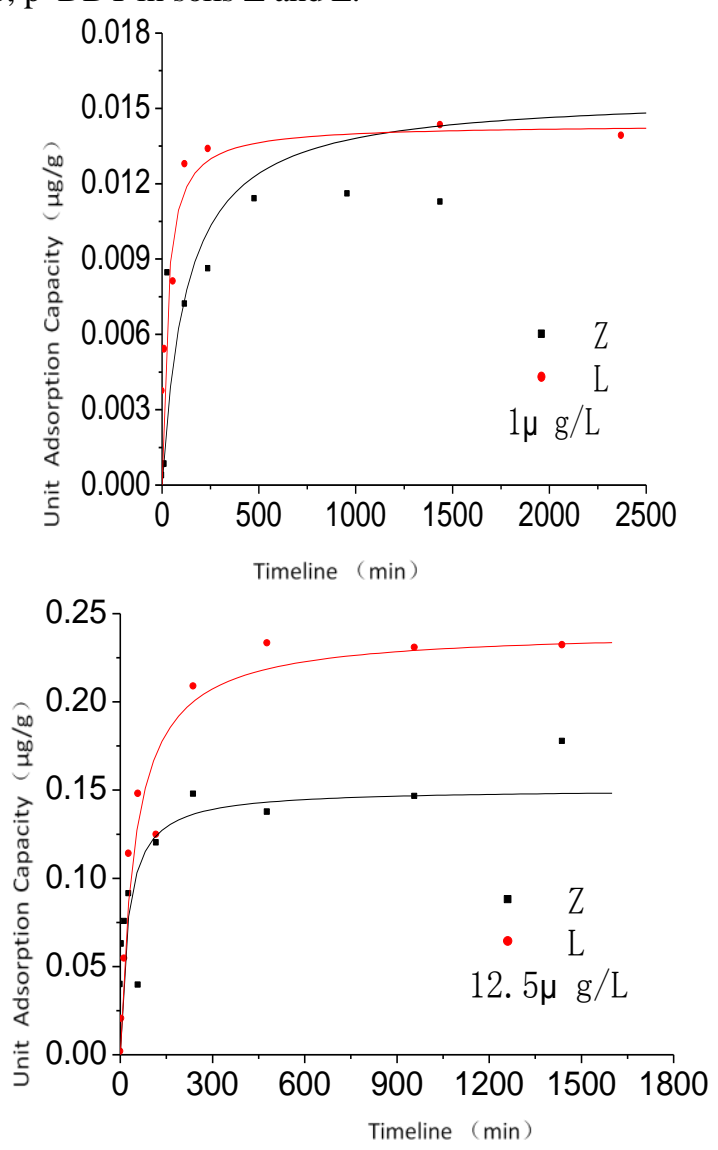

Figure 1. Kinetic process of adsorption of $\alpha-\mathrm{BHC}$ in two types of soils under two initial concentrations 

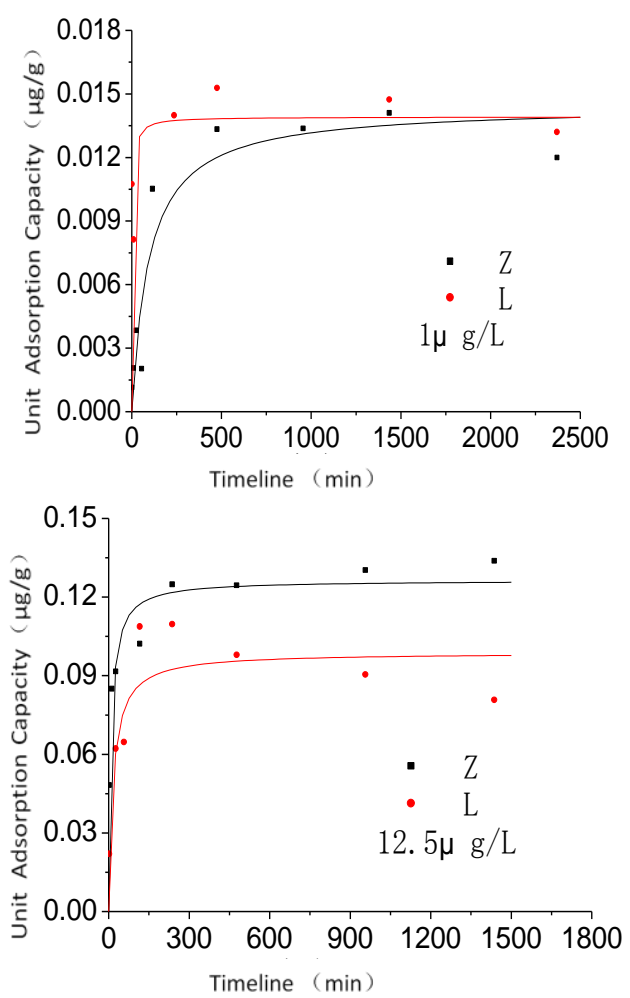

Figure 2. Kinetic process of adsorption of $\gamma$-BHC in two types of soils under two initial concentrations
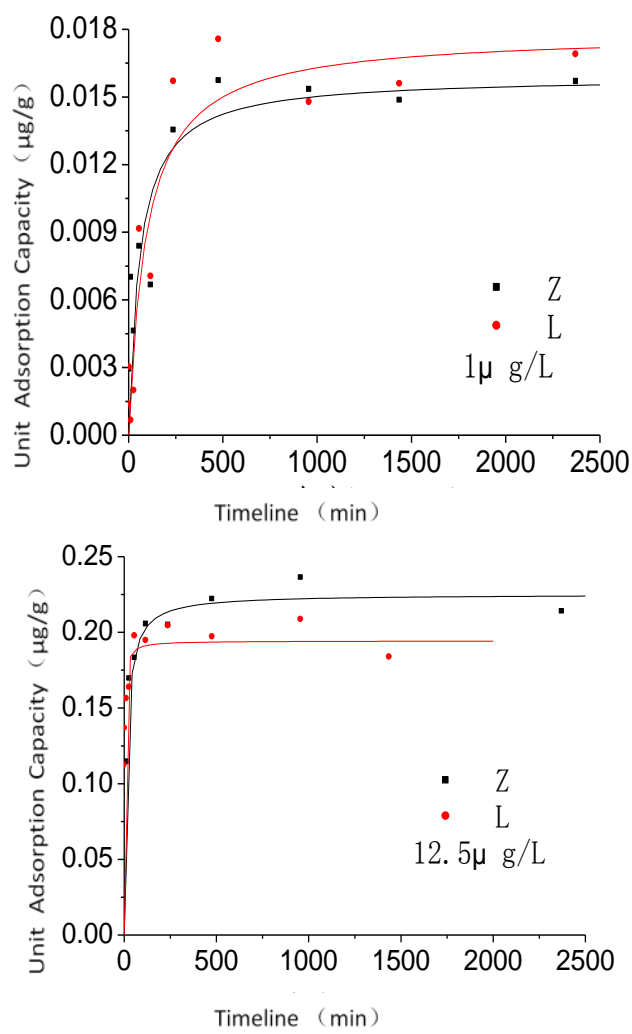

Figure 3. Kinetic process of adsorption of p,p'-DDT in two types of soils under two initial concentrations
It can be seen from the figure that the adsorption of BHC and DDT in soil can be divided into two processes, namely fast reaction phase and slow reaction phase. When the initial concentrations of $\alpha$-BHC and $\gamma$-BHC were $1 \mu \mathrm{g} / \mathrm{L}$ and $12.5 \mu \mathrm{g} / \mathrm{L}$, the adsorption capacity increased with time, and reached the equilibrium at about $4-8 \mathrm{~h}$. Within the first hour it can reach more than $50 \%$ of the equilibrium adsorption capacity, within $2 \mathrm{~h}$ it can reach more than $90 \%$ of the equilibrium adsorption capacity. However, due to the small amount of BHC adsorbed by the soil and the test errors, some data points showed irregular changes.

The adsorption of $\mathrm{p}$, $\mathrm{p}$-DDT in soils $\mathrm{L}$ and $\mathrm{Z}$ also follows this rule, and in the process of adsorption of $\mathrm{p}, \mathrm{p}$-DDT, the unit adsorption capacity of soil sample $\mathrm{L}$ and soil sample $\mathrm{Z}$ was similar when the kinetic adsorption process reached equilibrium. And the adsorption rate of the soil sample in the high concentration of adsorption solution was slightly faster than that of the low concentration solution.

In addition, the sorption / desorption processes actually occur simultaneously during the adsorption (desorption) of organic matter, and the adsorption / desorption twocompartment model can describe both reversible processes well. In addition to the Langmuir equation described above, experimental data are also described using a modified twocompartment model based on a first order kinetic process.

The adsorption of $\mathrm{p}, \mathrm{p}$-DDT in soils $\mathrm{L}$ and $\mathrm{Z}$ also follows this rule, and in the process of adsorption of p, p-DDT, the unit adsorption capacity of soil sample $\mathrm{L}$ and soil sample $\mathrm{Z}$ was similar when the kinetic adsorption process reached equilibrium. And the adsorption rate of the soil sample in the high concentration of adsorption solution was slightly faster than that of the low concentration solution.

TABLE II. THE FITTING RESUlts OF THE MODIFIED TwO-COMPARTMENT MODEL OF THE ADSORPTION KINETIC DATA

\begin{tabular}{lllll}
\hline $\begin{array}{l}\text { chemical } \\
\text { compound }\end{array}$ & soil & $\begin{array}{l}\mathrm{K}_{\mathrm{ad}}\left(\mathrm{L}^{1} \mathrm{~kg}^{-}\right. \\
\left.{ }^{1} \cdot \mathrm{h}^{-1}\right)\end{array}$ & $\begin{array}{l}\mathrm{K}_{\mathrm{de}}\left(\mathrm{L} \cdot \mathrm{kg}^{-}\right. \\
\left.{ }^{1} \cdot \mathrm{h}^{-1}\right)\end{array}$ \\
\hline$\alpha-\mathrm{BHC}$ & $\mathrm{Z}$ & 0.2765 & 0.0128 & 0.9206 \\
$(1 \mu \mathrm{g} / \mathrm{L})$ & $\mathrm{L}$ & 0.2868 & 0.0064 & 0.9187 \\
$\alpha-\mathrm{BHC}$ & $\mathrm{Z}$ & 1.3532 & 0.0554 & 0.8678 \\
$(12.5 \mu \mathrm{g} / \mathrm{L})$ & $\mathrm{L}$ & 0.3099 & 0.0022 & 0.9544 \\
$\gamma-\mathrm{BHC}$ & $\mathrm{Z}$ & 0.1155 & 0.0029 & 0.9651 \\
$(1 \mu \mathrm{g} / \mathrm{L})$ & $\mathrm{L}$ & 1.1918 & 0.0203 & 0.8609 \\
$\gamma-\mathrm{BHC}$ & $\mathrm{Z}$ & 0.9046 & 0.0450 & 0.9023 \\
$(12.5 \mu \mathrm{g} / \mathrm{L})$ & $\mathrm{L}$ & 0.0870 & 0.0058 & 0.8091 \\
$\mathrm{p}, \mathrm{p}^{\prime}-\mathrm{DDT}$ & $\mathrm{Z}$ & 0.1567 & 0.0025 & 0.9158 \\
$(1 \mu \mathrm{g} / \mathrm{L})$ & $\mathrm{L}$ & 0.1352 & 0.0015 & 0.9587 \\
$\mathrm{p}, \mathrm{p}^{\prime}-\mathrm{DDT}$ & $\mathrm{Z}$ & 0.9067 & 0.0080 & 0.9624 \\
$(12.5 \mu \mathrm{g} / \mathrm{L})$ & $\mathrm{L}$ & 5.8943 & 0.0977 & 0.8039 \\
\hline
\end{tabular}

In addition, the sorption / desorption processes actually occur simultaneously during the adsorption (desorption) of 
organic matter, and the adsorption / desorption twocompartment model can describe both reversible processes well. In addition to the Langmuir equation described above, experimental data are also described using a modified twocompartment model based on a first order kinetic process.

According to the adsorption / desorption twocompartment model, ignoring the loss effect like volatilization during the experiment $(\mathrm{Kv}=0)$, it is considered that the adsorption / desorption is the first-order kinetics process and that the mud / water is well mixed, we can get:

$$
\begin{gathered}
\frac{d C_{W}}{d t}=-\left(K_{v}+K_{a d} \cdot S_{W}\right) \cdot C_{W}+K_{d e} \cdot S_{W} \cdot C_{W} \\
=K_{d e} \cdot S_{W} \cdot C s-K_{a d} \cdot S_{W} \cdot C_{W} \\
\frac{d C_{S}}{d t}=K_{a d} \cdot C_{W}-K_{d e} \cdot C_{S}
\end{gathered}
$$

in the boundary conditions $\mathrm{t}=0, \mathrm{Cw}=\mathrm{Cw}^{0} ; \mathrm{t}=\mathrm{t}, \mathrm{Cw}=$ Cw, we can get:

$$
C w=C w^{0} \times \frac{K_{d e}+K_{a d} \cdot S_{W} \cdot e^{\left(K_{d e}+K_{a d} \cdot S W\right) \cdot t}}{K_{d e}+K_{a d} \cdot S_{W}}
$$

where $\mathrm{C}_{\mathrm{W}}$ is the concentration of organochlorine pesticides $(\mu \mathrm{g} / \mathrm{L})$ in water at time $\mathrm{t}, \mathrm{C}_{\mathrm{W}}{ }^{0}$ is the initial concentration $(\mu \mathrm{g} / \mathrm{L})$ of organochlorine pesticides in water, $\mathrm{K}_{\mathrm{de}}$ is the desorption rate constant for organochlorine pesticides $(\mathrm{L} / \mathrm{kg}(\mathrm{Kg} / \mathrm{h})), \mathrm{K}_{\mathrm{ad}}$ is the adsorption rate constant $(\mathrm{L} /(\mathrm{kg} \cdot \mathrm{h}))$ of organochlorine pesticides in soil, $\mathrm{S}_{\mathrm{W}}$ is the ratio of mud to water ( $\mathrm{kg} / \mathrm{L}, 0.05$ in this study). The adsorptive / desorbing rate constants were calculated by the above changes of the concentration of organochlorine pesticides in the non-linear fitted adsorption solution. The results are shown in Table II. The fitted r-values ranged from 0.8039 to 0.9651 , indicating that the modified twocompartment model could well describe the adsorption process of organochlorine pesticides.

\section{CONCLUSION}

The experimental study on the adsorption kinetics of organochlorine pesticides BHC and DDT in the indoor simulated soil showed the following conclusions: The results showed that the adsorption of BHC and DDT in the tested soils were well consistent with the Langmuir kinetics equation. Adsorption can be divided into two processes, namely, rapid reaction phase and slow reaction phase. At the initial stage of reaction, the amount of adsorption increased with time and basically reached equilibrium at about $4 \sim 8 \mathrm{~h}$. The equilibrium adsorption capacity was more than $50 \%$ in the first hour and above $90 \%$ in the second hour. In addition, the sorption and the desorption process actually occur simultaneously during the adsorption or desorption of organic matter. The adsorption / desorption twocompartment model can describe the two reversible processes at the same time. The fitted value $r$ is from 0.8039 to 0.9651 .

\section{ACKNOWLEDGMENTS}

This work was sponsored by Project 201434 supported by the transportation Department of Jilin province, whose support the authors gratefully acknowledge.

\section{REFERENCES}

[1] Li Kebin, Xu Zhongjian, Liu Weiping. The Research Progress on Soil's Absorption/Desorption of Pesticide and Effects on Bioavailability. Environmental Pollution Treatment Technology and Equipment. 2002, 3(4); 18 24.

[2] Barry, G., Oliver. And Karen, D. Nicol., Environ. Sci.Technol., 1982, 16: $532 \sim 536$

[3] Gao Taizhong, Huang Qunxian, etc. The Experiment and Reseach on Organic Pollutant's Migration and Transfer in Vadose Zone. Environmental Pollution Treatment Technology and Equipment. 2004, $5(2) ; 42 \sim 45$.

[4] Li Xueyuan. Soil Chemistry [M]. Beijing: Higher Education Press, 2001. 398-402.

[5] Editorial committee of the State environmental protection administration Water and Wastewater Monitoring Analysis Method [M]. Beijing: China Environmental Science Press, 1989, 156-159.

[6] Shan Zhengjun, Zhu Zhonglin. Transformation of Aldicarb of Three Sorts of Pesticides in Soil. Journal of Ecology and Rural Environment. 1994, 10(4); 30-33

[7] Li Xuqian, Shang Shubo, Lin Yaju, etc. The Measurement of the Hydrochemistry Mobility of the Oil Pollutant in the Aeration Zone. Journal of Jilin University (Earth Science Edition), 2005, 35(4); 501504.

[8] Yang Dawen, Yang Shixiu, Zhu Zhonglin. Preliminary Research on Pesticides' Migration in Soil and Influencing Factors. Journal of Soil, 1992, 29 (4); 383-391.

[9] Kang Yuehui, Sheng Guoying, Fu Jiamo. Vertical Distribution Characteristics of Organochlorinated Pesticides in Sediment Core from Macao Estuary, Pearl River Delta. Chinese Journal of Environmental Science, 2001, 22(1); 81-85.

[10] Stanislaw Maciejewski. Numerical and experimental study of solute transport in unsaturated soil. J of Contaminant Hydrology, 1993, 14: 193 206.

[11] Kookana RS, RD Schuller, LAG Aylmore. Simulation of simazine transport through soil columns using time-dependent sorption data measured under flow conditions. J of Contaminant Hydrology, 1993, $14: 93 \sim 115$ 